\title{
CORRESPONDENCE Recombinant vaccine containing an RBD-Fc fusion induced protection against SARS-CoV-2 in nonhuman primates and mice
}

\author{
Shihui Sun ${ }^{1}$, Lei $\mathrm{He}^{1}$, Zhongpeng Zhao ${ }^{1}$, Hongjing Gu${ }^{1}$, Xin Fang ${ }^{2}$, Tiecheng Wang ${ }^{3}$, Xiaolan Yang ${ }^{1}$, Shaolong Chen ${ }^{1}$, Yongqiang Deng ${ }^{1}$, \\ Jiangfan $\mathrm{Li}^{1}$, Jian $\mathrm{Zhao}^{4}$, Liang $\mathrm{Li}^{3}$, Xinwang $\mathrm{Li}^{5}$, Peng $\mathrm{He}^{2}, \mathrm{Ge} \mathrm{Li}^{6}$, Hao $\mathrm{Li}^{1}$, Yuee Zhao ${ }^{1}$, Chunrun Gao ${ }^{5}$, Xiaoling Lang ${ }^{4}$, Xin $\mathrm{Wang}^{5}$, \\ Guoqiang $\mathrm{Fei}^{5}$, Yan $\mathrm{Li}^{6}$, Shusheng Geng ${ }^{4}$, Yuwei Gao ${ }^{3}$, Wenjin Wei ${ }^{5}$, Zhongyu $\mathrm{Hu}^{2}$, Gencheng Han ${ }^{6}$ and Yansong Sun ${ }^{1}$ \\ Cellular \& Molecular Immunology (2021) 18:1070-1073; https://doi.org/10.1038/s41423-021-00658-z
}

The novel coronavirus SARS-CoV-2 has infected more than 104 million individuals and resulted in more than 2.2 million deaths worldwide as of February 7, 2021 (https://covid19.who.int). The COVID-19 pandemic highlights the need for safe and effective vaccines against SARS-CoV-2 infection. Several licensed vaccines and multiple vaccine candidates currently in clinical trials have shown different strengths and weaknesses (https://www.who.int/ emergencies/diseases/novel-coronavirus-2019/covid-19-vaccines). Herein, we report the pilot-scale production of a recombinant subunit vaccine (RBD-Fc Vacc) with the receptor-binding domain of the SARS-CoV-2 S protein fused with the Fc domain of human IgG1. The immunogenicity of the SARS-CoV-2 RBD, which is located in the S1 subunit and is crucial in mediating viral entry into host cells by binding to the ACE2 receptor, has been determined to induce neutralizing antibodies without evident antibody-dependent enhancement effects ${ }^{1}$ and can protect animals against SARS-CoV-2 infection ${ }^{2}$. The Fc fusion protein has been recently used as an important backbone for drug development due to its advantages of rapid purification, a relatively long half-life, and the ability to increase the immunogenicity of target antigens $^{3}$. In addition, Fc promotes the correct folding of the fusion protein and enhances binding to antigen-presenting cells ${ }^{4}$. We have previously developed Fc-fused protein vaccines against MERS, SARS-CoV, and H5N1 influenza and found that Fc-fused proteins are more immunogenic than those lacking fused $\mathrm{Fc}^{5,6}$. The advantages of recombinant protein vaccines, including safety (no viral genome integration, which ensures safe handling) and higher cost efficiency than other types of vaccines ${ }^{7}$, make them competitive vaccine candidates. In the present study, we developed a recombinant vaccine containing an RBD-Fc fusion (RBD-Fc Vacc), which is currently being assessed in randomized controlled phase I/II human clinical trials. In this study, the data showed the efficacy of RBD-Fc Vacc in protecting against SARSCoV-2 infection in nonhuman primates and mice.

In this study, the dimer-based RBD-Fc Vacc against SARS-CoV-2 was first developed by fusing the RBD (aa 331-524) with the human $\operatorname{lgG} 1$ Fc fragment. The dimer structure predicted by protein structure prediction server version 3.0 is shown in Fig. 1a (left panel). Two RBD domains were fused through the FC fragment to form the Y-shaped structure. The RBD-Fc fusion protein was expressed in mammalian $\mathrm{CHO}$ cells, and the antigenicity and dimer conformation were identified by western blot analysis using antiserum from recovered COVID-19 patients and a commercial antibody, respectively (Fig. 1a, right panel). Three N-glycosylation sites on asparagines, one O-glycosylation on a serine, and one O-glycosylation site on a threonine were identified using mass spectrometry. These identified glycosylation sites were further mapped to the complex structure of SARSCoV-2 RBD-Fc bound to ACE2 predicted by the ZDOCK server. The glycosylation sites were located in the RBD core subdomain and were found to be distant from the area bound to ACE2 (Fig. 1b), indicating that the glycopeptides may not interfere with receptor recognition. The binding of the RBD-Fc protein with ACE2 was then confirmed via surface plasmon resonance (SPR; Biacore). The RBD-Fc protein bound to the hACE2 receptor with much higher avidity than the RBD-His monomer (74.13 nM vs. $8.26 \mathrm{nM}$ ), indicating the improved conformation of our RBD-FC protein (Supplementary Fig. S1a). The immunogenicity of the RBD-Fc and RBD-His proteins was evaluated in BALB/c mice after two immunizations using aluminum as the adjuvant. Compared to serum from RBD-His-vaccinated mice, serum from RBD-FC vaccinated mice showed significantly higher RBD-specific $\lg$ antibody titers and live virus-neutralizing antibody titers (Supplementary Fig. S1b). These data suggested that the RBD-Fc fusion protein was more antigenic than the monomeric RBD protein.

We then evaluated the immunogenicity of RBD-Fc Vacc in Macaca fascicularis ( $n=5 /$ group) via intramuscular administration of $20 \mu \mathrm{g}$ or $40 \mu \mathrm{g}$ RBD-Fc Vacc or PBS control. The schedules for sample collection and examination are shown in Fig. 1c. High levels of RBD-specific immunoglobulin G (IgG) were rapidly induced in the serum of RBD-Fc Vacc-immunized Macaca fascicularis macaques, with median peaks of $1 / 17399$ in the 20 $\mu \mathrm{g}$ group and $1 / 41900$ in the $40 \mu \mathrm{g}$ group on day 28 (Fig. 1d). There was a much higher NT50 in sera from Macaca fascicularis macaques immunized with three doses of RBD-Fc Vacc (day 42)

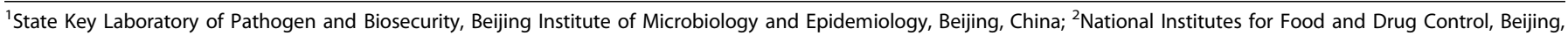

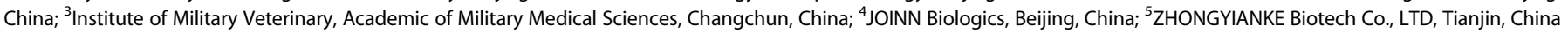
and ${ }^{6}$ Beijing Institute of Basic Medical Sciences, Beijing, China

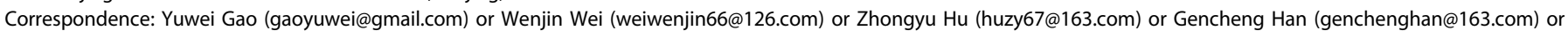
Yansong Sun (yansongsunamms@126.com)

These authors contributed equally: Shihui Sun, Lei He, Zhongpeng Zhao, Hongjing Gu, Xin Fang, Tiecheng Wang, Xiaolan Yang, Shaolong Chen, Yongqiang Deng.
}

Received: 7 February 2021 Accepted: 9 February 2021

Published online: 17 March 2021 

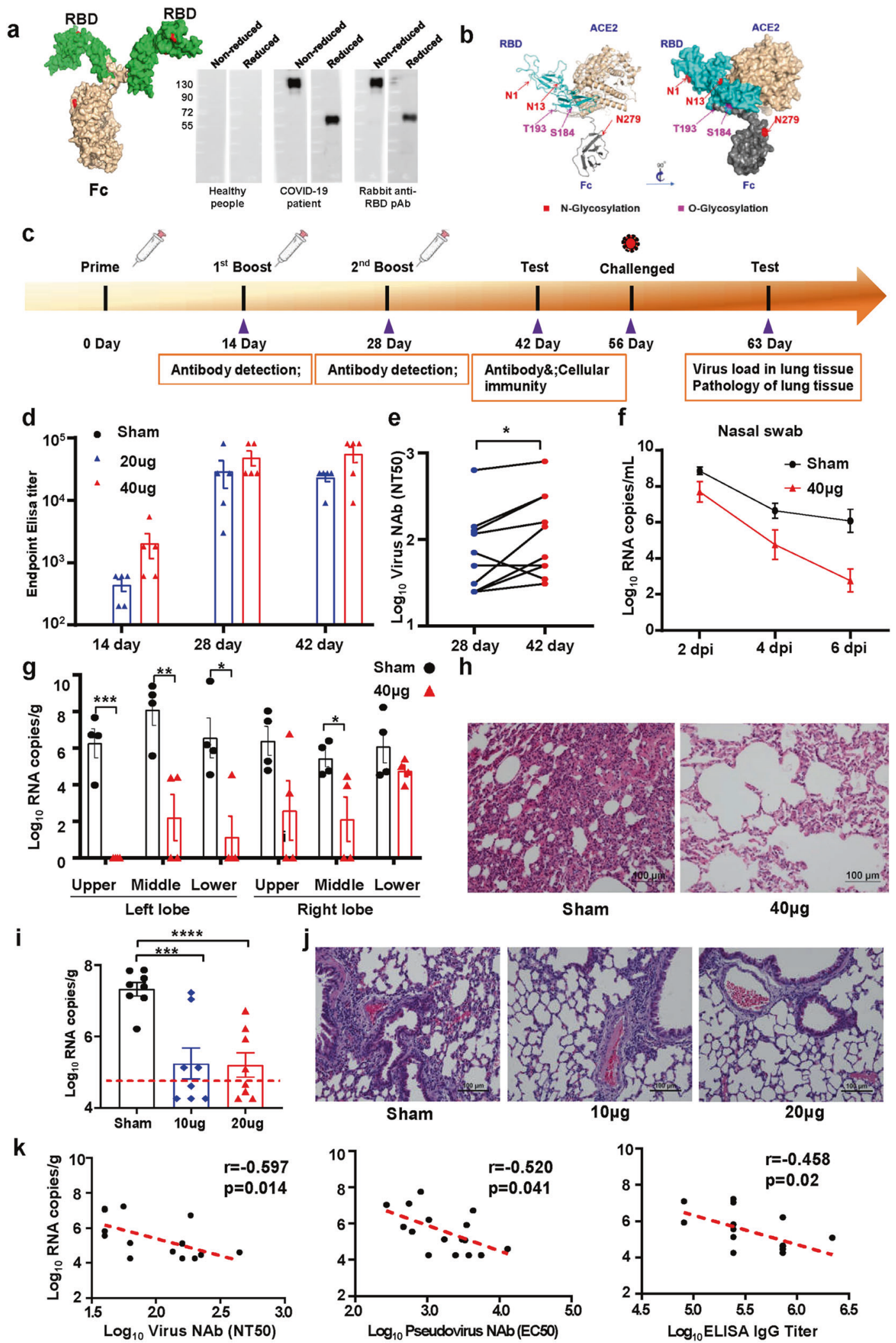

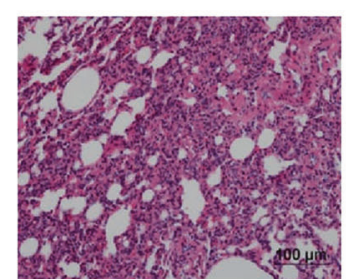

Sham

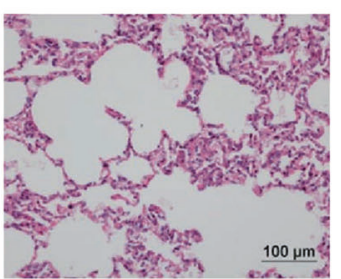

$40 \mu g$
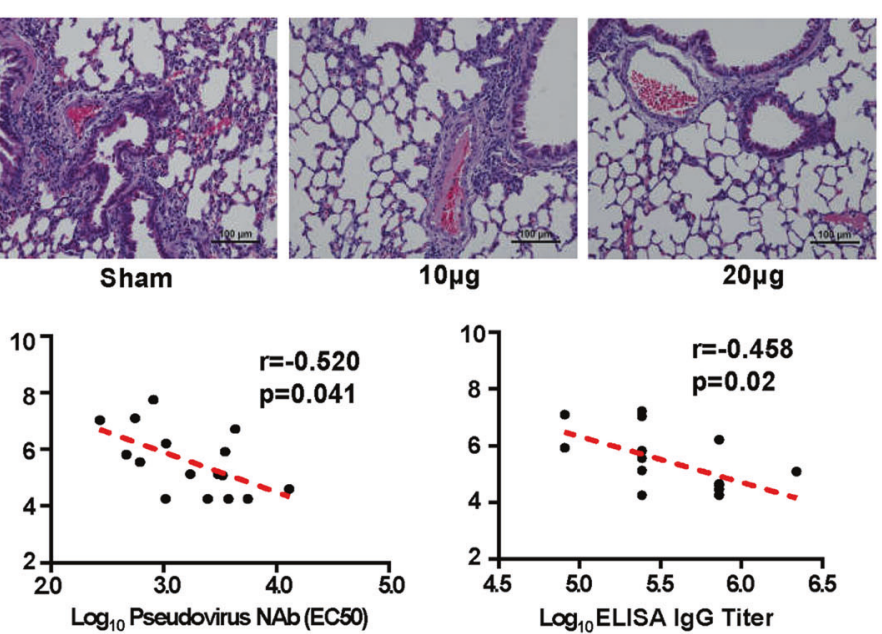

than in sera from macaques immunized with two doses of RBD-Fc Vacc (d28) (Fig. 1e), which indicated that immunization with three doses may induce much better protective responses. When the cross-neutralizing activity was analyzed, Macaca fascicularis sera post RBD-Fc vaccination showed similar neutralizing capability against three SARS-CoV-2 strains with the same RBD sequence as the one in our RBD-Fc vaccine ${ }^{8}$ (Supplementary Fig. S2a). More importantly, immunization with the RBD-Fc fusion protein protected $\mathrm{BALB} / \mathrm{C}$ mice against infection with the SARS-CoV-2 adapted virus MASCp6, which contains the N501Y mutation ${ }^{9}$, a 
Fig. 1 SARS-CoV-2 RBD-Fc Vacc induced a protective immune response in nonhuman primates and mice. a Two RBD domains were fused through the Fc fragment to form the Y-shaped structure via protein structure prediction server version 3.0 (left panel). The RBD-Fc protein expressed in $\mathrm{CHO}$ cells was identified by western blot analysis under reducing and nonreducing conditions using both antiserum from recovered COVID-19 patients and a commercial antibody (right panel). b N-glycosylation and O-glycosylation sites were identified by mass spectrometry. Docking between ACE-2 and RBD-Fc was predicted by the ZDOCK server. An overview of the glycosylation sites is shown based on the solved complex structure of SARS-CoV-2 RBD-Fc bound to ACE2. The identified sites, red for N-glycosylation and purple for O-glycosylation, are shown as spheres and labeled. The right panel (surface representation) was generated by rotating the structure in the left panel (cartoon representation) around a vertical axis by approximately $90^{\circ}$ (lower panel). c Schematic diagram of immunization, sample collection and challenge schedule. $\mathbf{d}$ Macaca fascicularis macaques $(n=5)$ were immunized on days 0,14 , and 28 with $20 \mu \mathrm{g}$ and $40 \mu \mathrm{g}$ doses of RBD-Fc Vacc or with PBS, and serum was collected at the indicated times. The level of SARS-CoV-2 RBD-specific IgG was examined by ELISA. e Neutralizing antibodies were determined by a microneutralization assay using SARS-CoV-2 (NT50). $\mathbf{f}$ Viral load of nasal specimens collected from the inoculated immunized Macaca fascicularis $(n=4)$ on day 2, day 4 and day 6 post infection were monitored. $\mathbf{g}$ Viral loads in tissue from various lobes of the lung in inoculated Macaca fascicularis macaques $(n=4)$ on day 7 post infection were measured. $\mathbf{h}$ Histopathological analysis of lungs from inoculated Macaca fascicularis macaques $(n=4)$ on day 7 post injection. Lung tissues were collected and stained with hematoxylin and eosin. i hACE2-Tg mice were immunized three times as described in the materials and methods $(n=8)$. Postinfection viral loads in lung tissue from inoculated hACE2-Tg mice were measured. The dashed lines indicate the detection limit of the assay. j Histopathological analysis of lungs from all inoculated hACE2-Tg mice post injection. $\mathbf{k}$ Correlations of SARS-CoV-2 NAb titers (left panel), pseudovirus NAb titers (center panel), and RBD protein-specific lgG titers (right panel) in hACE2-Tg mice prior to challenge with an amount of virus equivalent to the log peak mRNA copies/g in lungs measured after challenge. The red lines indicate the best-fit relationship between these variables. The $\mathrm{P}$ and $\mathrm{R}$ values were calculated by two-sided Spearman rank correlation tests. In d)-k), all data are presented as the mean \pm SEM of two independent experiments. ${ }^{*} p<0.05 ;{ }^{* *} p<0.01 ;{ }^{* * *} p<0.001$

key mutation that increases the binding affinity to human and murine ACE2 and has been found in the epidemic strain B.1.1.7 $7^{10}$. These data suggested that the RBD-Fc-based vaccine could induce broader neutralizing activity than current vaccines. In addition, the NT50 in sera from nonhuman primates receiving RBD-Fc Vacc immunization was comparable to that in sera from 23 convalescent COVID-19 patients (Supplementary Fig. S2b). Collectively, these results demonstrated that three immunizations with RBD-Fc Vacc induced high levels of neutralizing antibodies against SARSCoV-2 in nonhuman primates.

To explore the cellular immune response induced by RBD-Fc Vacc, an IFN- $\gamma$ ELISPOT assay and flow cytometry were performed in Macaca fascicularis on d42. There were comparable S1- or RBDspecific IFN- $\gamma$ responses in splenocytes from nonvaccinated and RBD-Fc Vacc-vaccinated Macaca fascicularis macaques (Supplementary Fig. S3a), and the percentages of S1-specific IL-4+CD4+ T cells, IL-4+CD8 + T cells, TNF-a+CD4+ T cells, and TNF-a+CD8+ T cells were comparable (Supplementary Fig. S3b), suggesting that RBD-Fc Vacc mainly induced a humoral immune response in vaccinated animals.

We next evaluated whether RBD-Fc Vacc induces protective immunity against SARS-CoV-2 infection in Macaca fascicularis macaques. Four weeks after the third injection of $40 \mu \mathrm{g} \mathrm{RBD-Fc}$ Vacc (d56), macaques were challenged with $10^{6} \mathrm{TCID} 50 / \mathrm{mL}$ SARSCoV-2 as follows: inoculation of $2 \mathrm{~mL}$ by the intratracheal route, $1 \mathrm{~mL}$ by the intranasal route and $0.2 \mathrm{~mL}$ by the intraocular route. The substantial fraction of viral RNA, which represents the input challenge virus, in nasal, throat, and anal swabs was examined at different times by quantitative reverse transcription PCR. Peak viral loads were observed at various time points post challenge. All control Macaca fascicularis macaques showed excessive copy numbers of viral genomic RNA in nasal (Fig. 1f), throat, anal (Supplementary Fig. S4) and lung swabs by days 2-6 post inoculation (Fig. $1 \mathrm{~g}$ and Supplementary Table S1), along with more severe interstitial pneumonia than vaccinated macaques (Fig. 1h). In contrast, all vaccinated Macaca fascicularis macaques were protected against SARS-CoV-2 infection, with much lower or no viral RNA copies and very mild histopathological changes in a few lobes of the lung. These data demonstrated that RBD-Fc Vacc induced a potent immune response, which protected Macaca fascicularis macaques against SARS-CoV-2 infection.

The protective efficiency of RBD-Fc Vacc against SARS-CoV-2 infection was also confirmed in hACE2-Tg mice using the same protocols as those used in Macaca fascicularis (Fig. 1c). In hACE2Tg mice, RBD-Fc Vacc immunization contributed to median reductions of $2.10 \log _{10}$ RNA copies/g and $2.13 \log _{10} \mathrm{RNA}$ copies/g in the $10 \mu \mathrm{g}$ and $20 \mu \mathrm{g}$ groups, respectively (Fig. 1i). All vaccinated animals showed very mild histopathological changes, while moderate interstitial pneumonia was observed in nonvaccinated controls (Fig. 1j). Finally, we analyzed the live virus neutralization antibody titers, pseudovirus neutralization antibody titers and IgG titers in hACE2-Tg mice on day 49. The results showed that all antibody titers were inversely correlated with the viral RNA titer in the lungs of hACE2-Tg mice (Fig. 1k). The vaccine-induced immune response and protection also showed an inverse correlation in Macaca fascicularis macaques, although statistical analysis could not be performed due to the limitation of sample size. These data demonstrated that the serum IgG titers and neutralizing antibody titers elicited by RBD-Fc may correlate with protection against SARS-CoV-2 infection.

In summary, we generated a new recombinant vaccine by fusing the SARS-CoV-2 RBD with the Fc fragment of human IgG1 and assessed the protective efficacy of this vaccine against SARSCoV-2 challenge in nonhuman primates (Macaca fascicularis) and mice. To the best of our knowledge, this Fc fusion protein-based vaccine is the first to be tested in clinical trials.

\section{ACKNOWLEDGEMENTS}

This article is in memory of Prof. Yusen Zhou for his contributions to the project conception and article design. We also thank X.D. Yu and J.J. Zhao for excellent technical and biosafety support. This work was supported by the National Key Plan for Scientific Research and Development of China (2020YFC0860100, 2020YFC0841401, 2016YFD0500306), the National Natural Science Foundation of China (82041006), and the National Science and Technology Major Project of China (No. 2017ZX10304402003001)

\section{AUTHOR CONTRIBUTIONS}

S.S., L.H., Z.Z., H.G., X.F., W.T., X.Y., Y.D., S.C., J.L., T.W., J.Z., L.L., X.L., P.H., G.L., H.L., C.G., X.L., C.W., X.W., and G.F. performed the experiments; S.S., L.H., H.G., Z.Z., Y.L., S.G., W.W., Z.H., and G.H. analyzed the data; Y.L. and Y.S. conceived the project. S.S., G.Y., H.G., Z.Z., G.H. Y.L., W.W., Z.H., and Y.S. supervised the study and wrote the manuscript with input from all coauthors.

\section{ADDITIONAL INFORMATION}

Supplementary information The online version contains supplementary material available at https://doi.org/10.1038/s41423-021-00658-z.

Competing interests: The authors declare no competing interests. 


\section{REFERENCES}

1. Quinlan, B. D. et al. The SARS-CoV-2 receptor-binding domain elicits a potent neutralizing response without antibody-dependent enhancement. 2020.2004.2010. 036418, https://doi.org/10.1101/2020.04.10.036418\%JbioRxiv (2021).

2. Yang, J. et al. A vaccine targeting the RBD of the S protein of SARS-CoV-2 induces protective immunity. Nature 586, 572-577 (2020).

3. Wieland, A. \& Ahmed, R. Fc receptors in antimicrobial protection. Curr. Top. Microbiol. Immunol. 423, 119-150 (2019).

4. Martyn, J. C. et al. Surface display of IgG FC on baculovirus vectors enhances binding to antigen-presenting cells and cell lines expressing Fc receptors. Arch. Virol. 154, 1129-1138 (2009).

5. $\mathrm{Li}, \mathrm{Y}$. et al. A recombinant protein containing highly conserved hemagglutinin residues $81-122$ of influenza $\mathrm{H} 5 \mathrm{~N} 1$ induces strong humoral and mucosal immune responses. Biosci. Trends 7, 129-137 (2013).
6. Du, L. et al. Receptor-binding domain of SARS-CoV spike protein induces long-term protective immunity in an animal model. Vaccine 25, 2832-2838 (2007).

7. Kaur, S. P. \& Gupta, V. COVID-19 vaccine: a comprehensive status report. Virus Res. 288, 198114 (2020).

8. Zhang, N. N. et al. A thermostable mRNA vaccine against COVID-19. Cell 182, 1271-1283 e1216 (2020).

9. $\mathrm{Gu}, \mathrm{H}$. et al. Adaptation of SARS-CoV-2 in BALB/c mice for testing vaccine efficacy. Science 369, 1603-1607 (2020).

10. McCallum, M. et al. N-terminal domain antigenic mapping reveals a site of vulnerability for SARS-CoV-2. bioRxiv, https://doi.org/10.1101/2021.01.14. 426475 (2021) 